\title{
Research on Influence of Soil Moisture Content of Farmland on Infiltration Model Parameters
}

\author{
Zhiwei Zheng ${ }^{1, *}$, Zhuozhuo Gao ${ }^{1}$ \\ 1*Department of Hydraulic Engineering, Tianjin Agricultural University, Tianjin 300384, China \\ ${ }^{1}$ Department of Hydraulic Engineering, Tianjin Agricultural University, Tianjin 300384, China
}

\begin{abstract}
In order to study the influence of the initial moisture content on the parameters of the infiltration model using an indoor soil column test method, and the relationship between the initial moisture content and each model parameter was analyzed by using the Green-Ampt model, the Kostiakov model, and the Horton model. The results show that there is a certain relationship between the initial water content and the parameters of the infiltration model. Based on comprehensive considerations, the Kostiakov model is the best surface irrigation infiltration model, and the Kostiakov model has the best effect when the observation time is not less than 80 minutes to simulate the soil infiltration process.
\end{abstract}

\section{INTRODUCTION}

The process of soil infiltration determines the amount of precipitation or irrigation water entering the soil, which is not only related to the amount of water supplied to the crop in the current season, but also to the amount of deep water used by the crop after water supply. The study of soil infiltration characteristics is of great significance in agricultural science and tehnology. Soil infiltration is affected by factors such as initial soil moisture content, soil texture, and water depth.

This article aims to analyze the effects of soils with different initial moisture content on infiltration model parameters for indoor soil column tests under changes in initial moisture content and cumulative water content. The purpose is to prove the qualitative relationship between the initial moisture content and the parameters of each infiltration model.

\section{Materials and methods}

\subsection{Experimental method}

The testers took soil from the Tianjin Agricultural College's farmland test base and selected soil at the same depth from the ground (to ensure the same soil texture). After air drying, rolling, and passing through a soil sieve with a $2 \mathrm{~mm}$ aperture, the purpose of uniform mixing is achieved. We assume that the moisture content of the soil after air-drying is $1.5 \%$, and the initial soil moisture content levels of the four soils are set at $6 \%$, which are $7.5 \%, 13.5 \%, 19.5 \%$, and $25.5 \%$, respectively. The soil column used in the test was formed by bonding a $7 \mathrm{~cm}-$ high base with three soil columns $20 \mathrm{~cm}, 20 \mathrm{~cm}$, and $30 \mathrm{~cm}$ in height, all of which were $10 \mathrm{~cm}$ in diameter.
Before the test, according to the assumption of air-dried soil moisture content, set soil moisture content, ideal soil dry weight, ideal water weight, actual soil dry weight, actual soil weight, soil column volume $(10 \mathrm{~cm}$ diameter, $60 \mathrm{~cm}$ high cylindrical volume), soil Bulk density $\left(1.4 \mathrm{~g} / \mathrm{cm}^{3}\right)$ calculate the amount of water to be added and the amount of soil to be taken, and measure them. Use a tray balance to weigh the required dry soil weight, place it in a large enough container, and mix the four groups of water and soil evenly. Then cover it with a plastic tarpaulin and keep it sealed. Before loading the soil column, use medical tape to mark every $5 \mathrm{~cm}$ to facilitate the measurement of the wetting front. After the soil moisture is evenly distributed, divide it into 12 layers $(5 \mathrm{~cm}$ each) according to the design bulk density $\left(1.4 \mathrm{~g} / \mathrm{cm}^{3}\right)$, weigh the soil weight added to each layer, and load it into the test soil column (the foundation is filled with sand).

Before the experiment, the soil column was irrigated so that the water level was $5 \mathrm{~cm}$ above the soil surface, and the time was counted when the water no longer dropped significantly. When the water surface is significantly lower than the $5 \mathrm{~cm}$ mark, start adding water so that the water surface reaches the mark, and record the time and amount of water added to measure the wetting front. The test ended after 12 hours.

\subsection{Analytical methods}

There are many formulas for estimating the infiltration rate, which can be divided into semi-theoretical and semi-empirical models and empirical models. The commonly used semi-theoretical and semi-empirical models are the Green-Ampt model and the Philip model. The more widely used empirical models are the Kostiakov model and the Horton model.

\footnotetext{
* Corresponding author: zhiwei35883@163.com
} 
The Green-Ampt infiltration formula, which is specifically expressed as

$$
t=\frac{1}{i_{c}} \cdot\left[I(t)-\frac{b}{i_{c}} \ln \left(1+\frac{I(t) \cdot i_{c}}{b}\right)\right]
$$

$b$ is the parameter of the Green-Ampt formula and $i_{c}$ is the stable infiltration rate. The soil infiltration characteristics can be calculated as long as the soil saturation conductivity and the suction force of wetting peak surface are obtained.

The Kostiakov infiltration model can effectively describe the infiltration process in the short-term range, but it is not suitable for long-term infiltration study. It is expressed as

$$
I(t)=K \tau^{a}+f_{0} \tau(m m)
$$

The three parameters of the Horton model are determined based on the measured data. It can present the infiltration of soil factors to the affect of infiltration.It is expressed as

$$
i(t)=i_{c}+\left(i_{0}-i_{c}\right) \cdot e^{-\beta t}
$$

and

$$
I(t)=i_{c} t-\frac{1}{\beta}\left(i_{0}-i_{c}\right) \cdot e^{-\beta t}+\frac{1}{\beta}\left(i_{0}-i_{c}\right)
$$

In the formula: $\mathrm{i}_{0}$ is the initial infiltration rate.

Philip's infiltration model is

$$
i(t)=\frac{1}{2} S t^{-1 / 2}+A
$$

and

$$
I(t)=S t^{1 / 2}+A t
$$

which is only applicable to the case of one-dimensional vertical infiltration of homogeneous soil.

The total infiltration water and infiltration speed were calculated based on the accumulated time, cumulative water addition and wetting front value recorded in the test, and the simulation values were calculated according to the three infiltration model formulas. Calculate the square error between the simulated value and the actual value, and use programming solve to minimize the square error to calculate the optimal parameters.

\section{Results and discussion}

\subsection{Effect of initial moisture content on infiltration rate and cumulative infiltration process}

After the test, the soil in the two soil boxes was taken out every $5 \mathrm{~cm}$ previously set, and the actual data of the four soil columns were obtained. The actual initial water contents of the four soil columns were $6.3 \%, 11.0 \%$, $14.5 \%, 19.6 \%$. Through the subsequent series of data analysis, the relationships shown in Fig.1 and Fig.2 are obtained. We can clearly find that the initial water content has similar effects on the infiltration rate and cumulative infiltration process. On the one hand, the soil column with an initial moisture content of $19.6 \%$ has the slowest change in infiltration rate and cumulative infiltration volume with time and is also the slowest when compared with the other three soil columns. However, a soil column with an initial water content of $6.3 \%$ has the fastest infiltration rate and cumulative infiltration volume over time and the fastest comparison with the other three soil columns. In the longitudinal comparison, the soil columns with different initial moisture contents showed an increasing trend with time. On the other hand, when the four soil columns are compared horizontally, with the increase of the initial water content, their infiltration rate and cumulative infiltration rate change more slowly with time, and they gradually decrease. In addition, the maximum and minimum values of infiltration rate and cumulative infiltration water were obtained in two soil columns with an initial water content of $6.3 \%$ and an initial water content of $19.6 \%$, respectively.

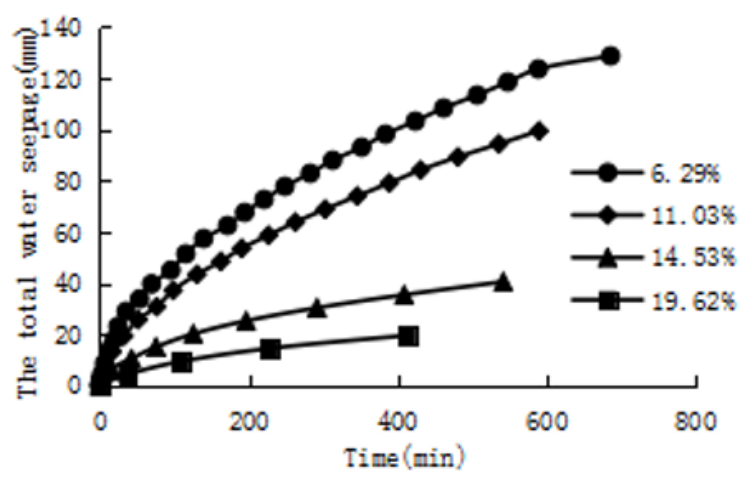

Fig. 1. Relationship between infiltration volume and time at different initial moisture contents.

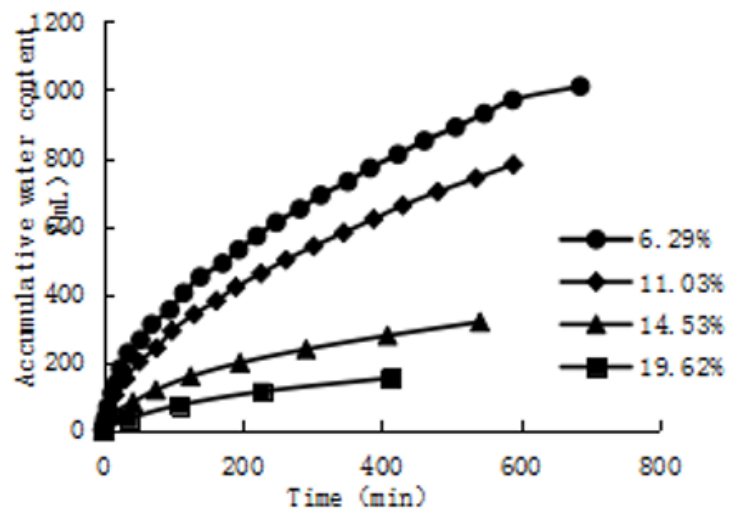

Fig. 2. Relationship between cumulative water addition and time at different initial water cuts.

\subsection{Effect of initial moisture content on parameters of infiltration model}

\subsubsection{Effect of initial water content on parameters of Green-Ampt infiltration model}

The actual cumulative time obtained through experiments is compared with the simulated cumulative time calculated by formula (1), then sum the square of the difference between them, and solve it in excel, and minimize the sum by changing the parameter values. The 
data obtained from the four soil columns were subjected to the same operation, and the relationship between the different initial moisture content and the parameters $b$ under this formula was obtained. The results are shown in Fig. 3:

\section{b}

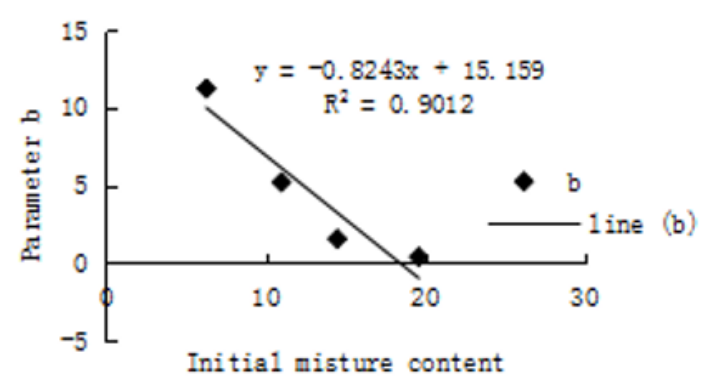

Fig. 3. Influence of different initial moisture content on parameter $b$

It can be seen from Fig. 3. that the parameter $b$ has a significant linear negative correlation with the initial water content, and the correlation coefficient reaches 0.9012 . That's means the parameter $\mathrm{b}$ decreases with the increase of the initial water content.

\subsection{Effect of initial moisture content on parameters of Kostiacov infiltration model}

The actual cumulative infiltration amount obtained through experiments is compared with the actual cumulative infiltration amount calculated by formula (3), then the square of the difference between them is summed, and use the same method described above to minimize the sum. Combining the four soil columns, we can see that fo is always 0 , so we don't consider the effect on $\mathrm{f} 0$, and the experimental results show that the initial water content has little effect on the parameter a. Therefore, the influence on parameter a can be ignored in the analysis, and parameter a can be regarded as a constant. The relationship between the parameter $\mathrm{K}$ and the initial water content is shown in Fig. 4:

\section{$\mathrm{K}$}

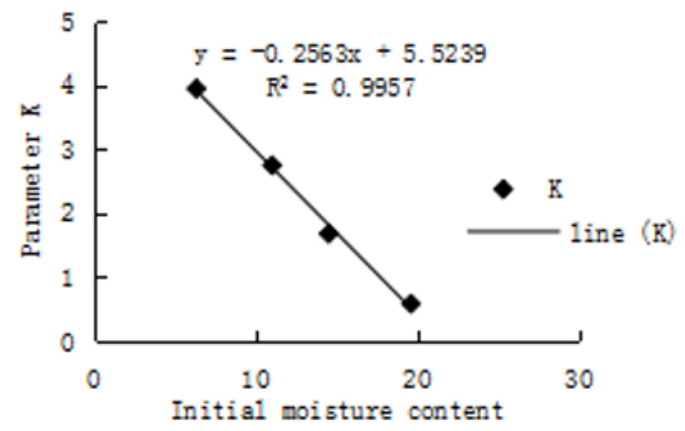

Fig. 4. Influence of different initial moisture content on parameter ic.

It can be obtained from Fig. 4. that the parameter K has a significant linear negative correlation with the initial moisture content, and the correlation coefficient is 0.9957 . That is means the parameter $\mathrm{K}$ decreases as the soil moisture content increases.

\subsection{Effect of initial water content on parameters of Horton infiltration model}

The actual cumulative infiltration amount obtained through experiments is compared with the actual cumulative infiltration amount calculated by formula (5), then the square of the difference between them is summed, and use the same method described above to minimize the sum. Combining 4 soil columns, we can get the influence of different initial water content on the parameters ic, i0 and $\beta$ under this formula. The results are shown in Fig. 5, Fig. 6, and Fig. 7:

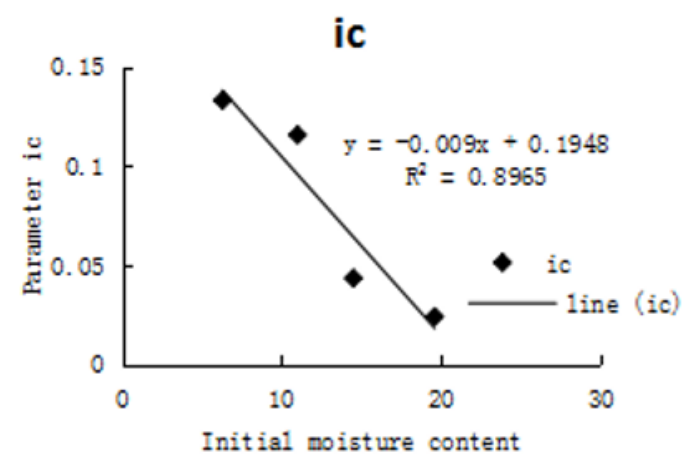

Fig. 5. Influence of different initial moisture content on parameter ic.

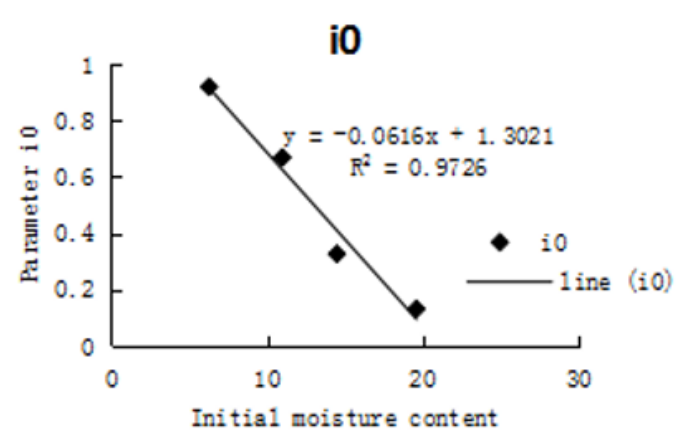

Fig. 6. Influence of different initial moisture content on parameter i0.

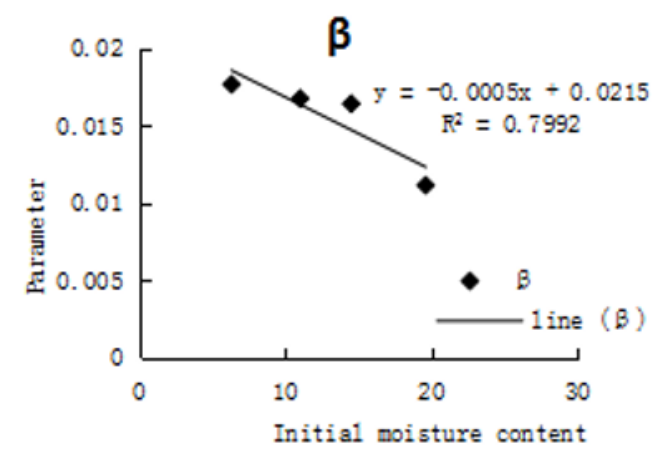

Fig. 7. Influence of different initial moisture content on parameter $\beta$. 
From Fig. 5, Fig. 6, and Fig. 7, the parameters ic, i0, and $\beta$ are significantly negatively correlated with the initial moisture content. Among them, the absolute value of the slope of the image in Fig. 6 is the largest, followed by Fig. 5, and finally Fig. 7, and the correlation coefficient also shows the same law. That is means the decreasing trend of $\mathrm{i} 0$ with the increase of the initial water content is the most obvious, and the decreasing trend of $\beta$ is the second, and the decreasing trend of $\beta$ is the last.

\subsection{Influence of initial water content on parameters of Philip infiltration model}

The actual cumulative infiltration amount obtained through experiments is compared with the actual cumulative infiltration amount calculated by formula (5), then the square of the difference between them is summed, and use the same method described above to minimize the sum. Combining 4 soil columns, we can get the influence of different initial moisture content on the parameters $\mathrm{S}$ under this formula. The results are shown in Fig. 8 :

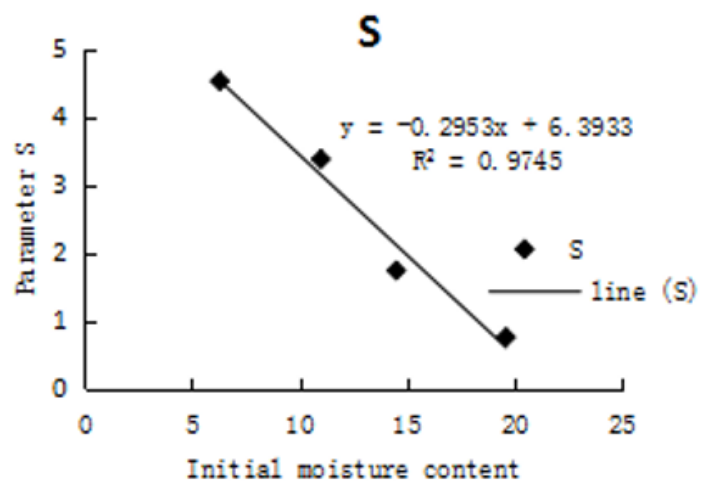

Fig. 8. Effect of different initial moisture content on parameter $\mathrm{S}$.

It can be obtained from Fig. 8 that the parameter $\mathrm{S}$ and the initial moisture content have a significant linear negative correlation, and the correlation coefficient is 0.9745. That's means the parameter $\mathrm{S}$ decreases as the initial moisture content increases.

\section{Conclusion}

In the three models, most of the affected parameters decrease with increasing initial water content. According to the fitting effect, the Kostiacov infiltration model formula is better, because there is only one affected parameter, and the linear correlation coefficient between this parameter and the initial water content is the largest, and the effect of the initial water content can be better observed.

\section{Acknowledgment}

Funds for this research was provided by Tianjin Science and Technology Support Key Project(18YFZCSF00650), Tianjin Science and Technology Commissioner Project (19JCTPJC58300), Tianjin Wuqing Science and Technology Development Project (WQKJ201804), Tianjin Agricultural College Students' Innovation and Entrepreneurship Training Project (201910061099).

\section{References}

1. Xinyang Zhou et al.Study on adaptability of four infiltration formulas to farmland fluvo-aquic soil[J].Journal of Irrigation and Drainage, 2015, 34(8):86-100.

2. Wentao Fan et al.Characteristic analysis of interpolation parameters between Philip model and modified Green-Ampt model[J].Journal of Irrigation and Drainage, 2012, 31(2):73-77.

3. Chen Zeng et al.Effect of initial water content on vertical line source infiltration characteristics of soil[J].Journal of Agricultural Engineering, 2010, 26(1): 24-30.

4. Yanwei Fan et al.Improvement and verification of Green-Ampt infiltration model for sand layer soil[J]Journal of Agricultural Engineering, 2015, 31(5):93-99.

5. Yousheng Xiong et al.Comparative analysis of water infiltration models of purple soil in the Three Gorges reservoir area[J].Journal of Irrigation and Drainage, 2013, 32(1): 43-46.

6. Chuncheng Liu et al.Applicability of four infiltration models to infiltration law of waterrepellent soil[J].Journal of Agricultural Engineering, 2011, 27(5): 62-67.

7. Shiliang $\mathrm{Wu}$ et al.Spatial variability of soil characteristics and calibration factors of Philip infiltration formula[J].Journal of Drainage and Irrigation Machinery Engineering, 2014, 32(8):730736. 